\title{
MYTOXDATA: A TOXICOLOGICAL DATA MANAGEMENT FOR NATIONAL POISON CENTRE
}

\author{
Yulita Hanum P Iskandar ${ }^{1}$, Adilah Mohamed Ariff ${ }^{2}$, Rosman Ahmad ${ }^{2}$, Mohamed Isa Abd Majid ${ }^{2}$ and \\ Balamurugan Tangiisuran ${ }^{2}$ \\ ${ }^{1}$ Graduate School of Business, Universiti Sains Malaysia, 11800 USM, Penang, Malaysia \\ ${ }^{2}$ National Poison Centre, Universiti Sains Malaysia, 11800 USM, Penang, Malaysia ness
}

\author{
Corresponding author: Yulita Hanum P Iskandar \\ E-mail: yulita@usm.my
}

\begin{abstract}
The aim of the present paper is to evaluate the Malaysian Toxicological Database (MyToxData), a newly developed system for toxicological data management. MyToxData is a local system for collection of case reporting and toxicity statistics as well as its database of chemical products and toxicity information. An online questionnaire using JotForm was distributed among healthcare professionals in Malaysian hospitals. The participants were asked to perform tasks on MyToxData system before rating their satisfaction experience. The questionnaire was adopted from previous literature and comprised 12 items, each having a five-point scale that ranged from Strongly Disagree to Strongly Agree. The collected data were then analysed using the SPSS software package. Based on the results, the satisfaction testing showed a high satisfaction score for the login at $95.7 \%$ and case registration and toxicological/poisoning case notification at $65.2 \%$. The poisoning substance identification component received a $78.2 \%$ agreement on the usefulness with a very high acceptance of $91.3 \%$ on the treatment and clinical information retrieval made available on the system. One sample t-test found that all constructs for the satisfaction testing of MyToxData, namely, accuracy, content, ease of use, format and timeliness, were significant. The higher acceptance by healthcare professionals on the satisfaction testing of MyToxData has identified the effectiveness of the development and subsequent usage of a dedicated system for toxicological data management.
\end{abstract}

Keywords: Toxicological information system, National poison centre, Satisfaction, Healthcare professionals, Data management

\section{INTRODUCTION}

In November 1994, as per the directive of the Malaysian Cabinet, the National Poison Centre or Pusat Racun Negara (PRN) at Universiti Sains Malaysia was established. Its establishment as the nation's sole poison centre was a culmination of more than a decade of conceptualising, planning and implementing activities concerning drug and poison information known as Integrated Drug and Poison Information Services.

The PRN was primarily commissioned to function as a referral centre to enhance management and care of poisoned patients by providing information on poisons and antidotes availability and performing toxicology laboratory tests and analysis. Furthermore, the PRN is tasked to fulfil its public health mission in toxic surveillance, environmental health monitoring and poison education and prevention. The Drug and Poison Information Services (DPIS) is a unit in the PRN functioned to audit health information as well as upgrading and strengthening the handling of drug and poison information directed towards allied health professionals as well as the lay public. The DPIS mainly received calls concerning toxicological cases of a drug overdose, natural toxins and chemicals exposure.
In providing related information, the service officers simultaneously collect data on the demographic and clinical details of the poisoning case exposure for toxicovigilance and publication initiatives; hence, the centre has been referred for statistical data retrieval of toxicological cases in Malaysia when certain data from other sources are not easily accessible, are scattered and may be under-reported.

The PRN reported an increasing trend of a total of 39,088 poisoning exposure calls in 10 year period, i.e. from 2006 to $2015^{1}$. This number may not represent the total burden of poisoning exposures in Malaysia since the PRN is not a reporting agency and the data were collected only from enquiries made to the DPIS. Many years back, the PRN had collaborated to study hospital admission data from the Ministry of Health's Hospital Management Information System, where a total 21,714 toxicological cases admission in 3 year period, i.e. from 1999 to 2001, were studied ${ }^{2}$. This figure still outnumbered the number of cases referred to the PRN if we compare the figures in 3 years with the highest cases from 2006 to 2015. Another shortcoming is that the figures in 19992001 did not include non-admission cases and cases admitted to private healthcare facilities. Therefore, enhancing of data management and reporting of poisoning exposure in Malaysia with possible strategic initiatives and collaboration 
with relevant agencies and institutions are necessary.

Consultation by the DPIS is assisted by subscribed databases that are very costly, and recording of poisoning data was done manually via paper documentation and electronic entry. The previous system used was IPCS INTOX ${ }^{3}$ and the PRN's inhouse cloud storage data management. However, issues including sustainability, data reliability and validity, ease of use and multi-functionality may not be optimally addressed by previous documentation method. Over time, operating costs kept increasing, and there were times that the offline database required certain specifications of the computer's operating system and capacity. The DPIS is unique in its operation that can be done in a work-from-home set-up during certain operating hours, but well-equipped device and database are a vital requirement for each serving officer. The service is mainly operated by pharmacists, and human resource challenges are another factor that had impacted the operating costs. Needless to say, all these factors should be considered when dealing with change and challenges in operating cost, human resources and technology advancement. One of the efforts undertaken by the DPIS team was to develop a one-stop web-based system for toxicological/poisoning case notification, treatment and clinical information retrieval, poisoning substance identification and statistical data retrieval as an innovative contribution to maintaining the important service for the country.

Because of the toxicological data management issues in the country, the Malaysian Toxicological Database (MyToxData) system has been developed to enhance data collection processes and its management. In short, the system was developed to enhance the management of the toxicologyrelated services and to find solutions on the issues concerning the collection of toxicological data in Malaysia. Some researchers have suggested that satisfaction is one of the key factors leading to health information system success ${ }^{4}$ and the usability of interfaces can be considered as one of the factors that influence end-user satisfaction ${ }^{5}$. Therefore, the present paper aims to evaluate the satisfaction experience of healthcare professionals on the use of MyToxData system for its further enhancement with the goal of catering the practicality needs in a healthcare setting. The structure of the present paper is as follows. The intent of this article is to describe the poisoning issues and the proposed poisoning system, illustrate how the proposed system influence the health professionals and highlight the importance of integrating the data based on the public health infrastructure.

\section{METHODS}

Proposed MyToxData

MyToxData (see

Figure 1) is a local system for poisoning/toxicological case notification and statistical data retrieval as well as a database for chemical products and treatment and clinical information retrieval. It is a web-based database system developed by Universiti Sains Malaysia team consisting of the PRN, the Centre of Information, Communication and Technology and the Graduate School of Business, USM. The system targeting multiple designated users requires a login for security and data privacy. 


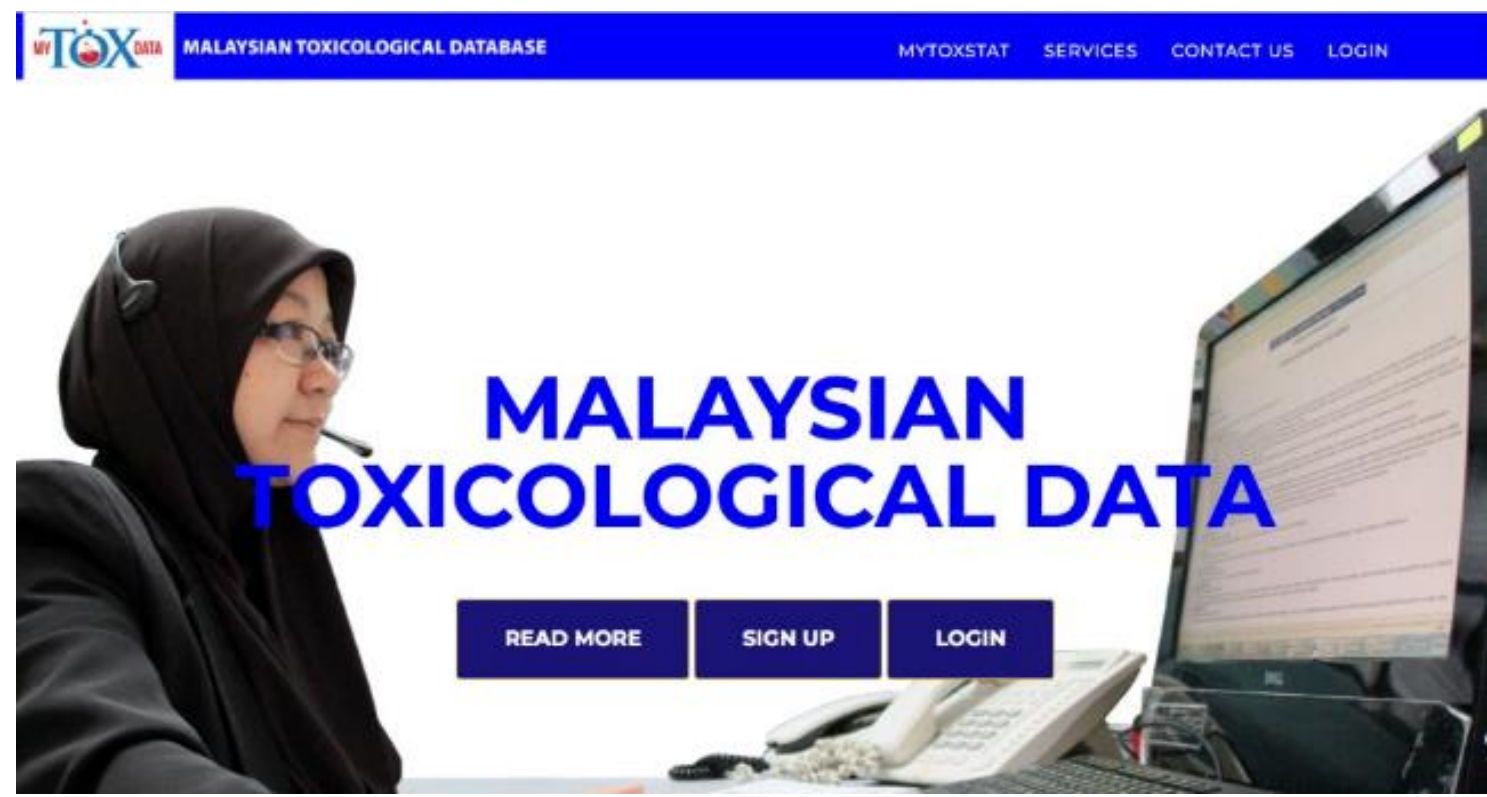

Figure 1: Main interface of the proposed system

Figure 2 shows the five main components of the system include (1) case registration, (2) toxicological/poisoning case notification, (3) poisoning substance identification, (4) treatment and clinical information retrieval and (5) statistical data retrieval.
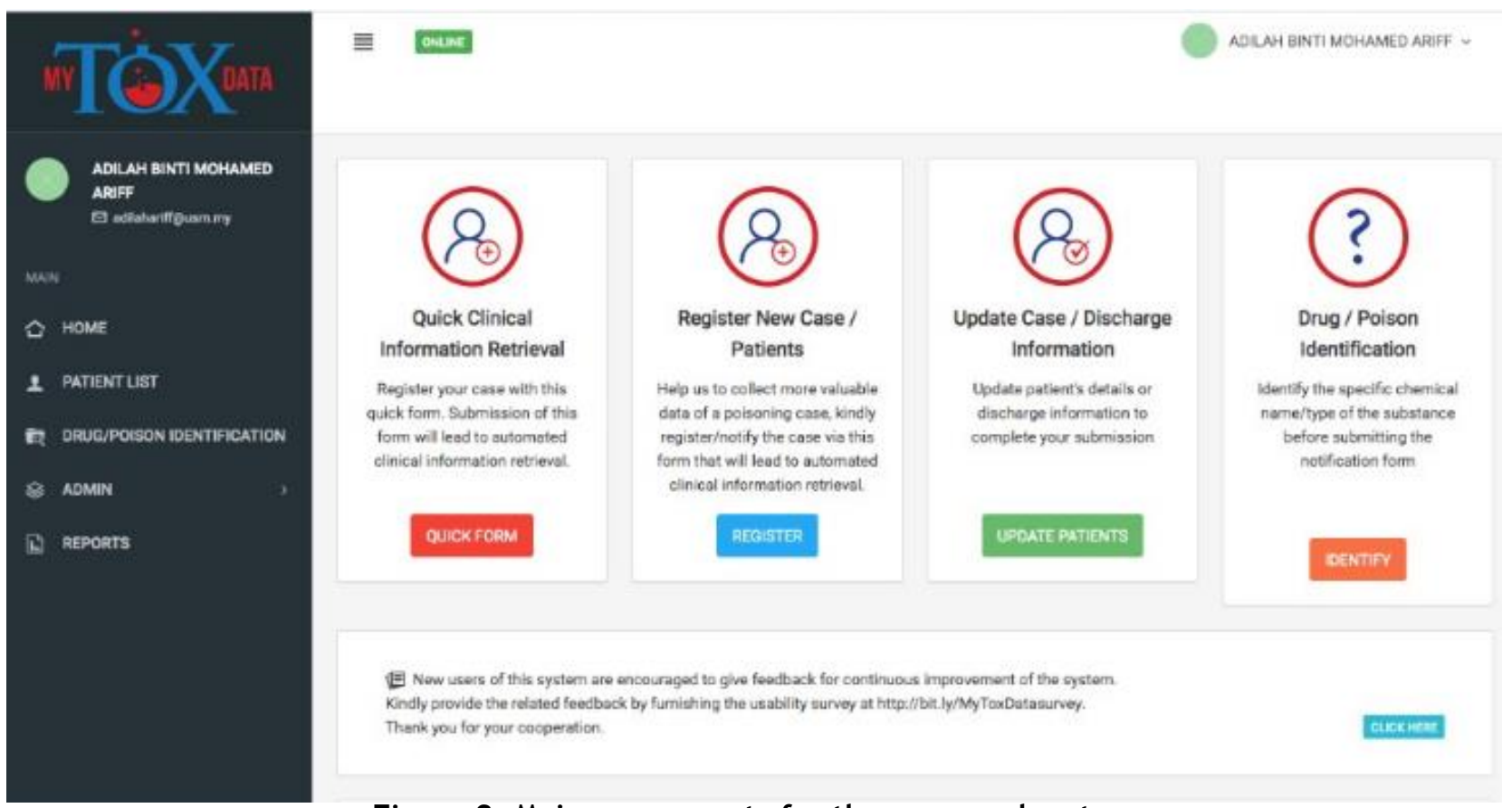

Figure 2: Main components for the proposed system

Login and case registration are part of the components of MyToxData system (see

Figure 3 ). The system provides a quick and easy case registration form to be filled by the healthcare professionals on the poisoning cases. The quick information is crucial as most of the poisoning cases notified to the PRN are from healthcare professionals. The essential information in the form had been validated through several interview sessions with healthcare professionals and PRN staff. 


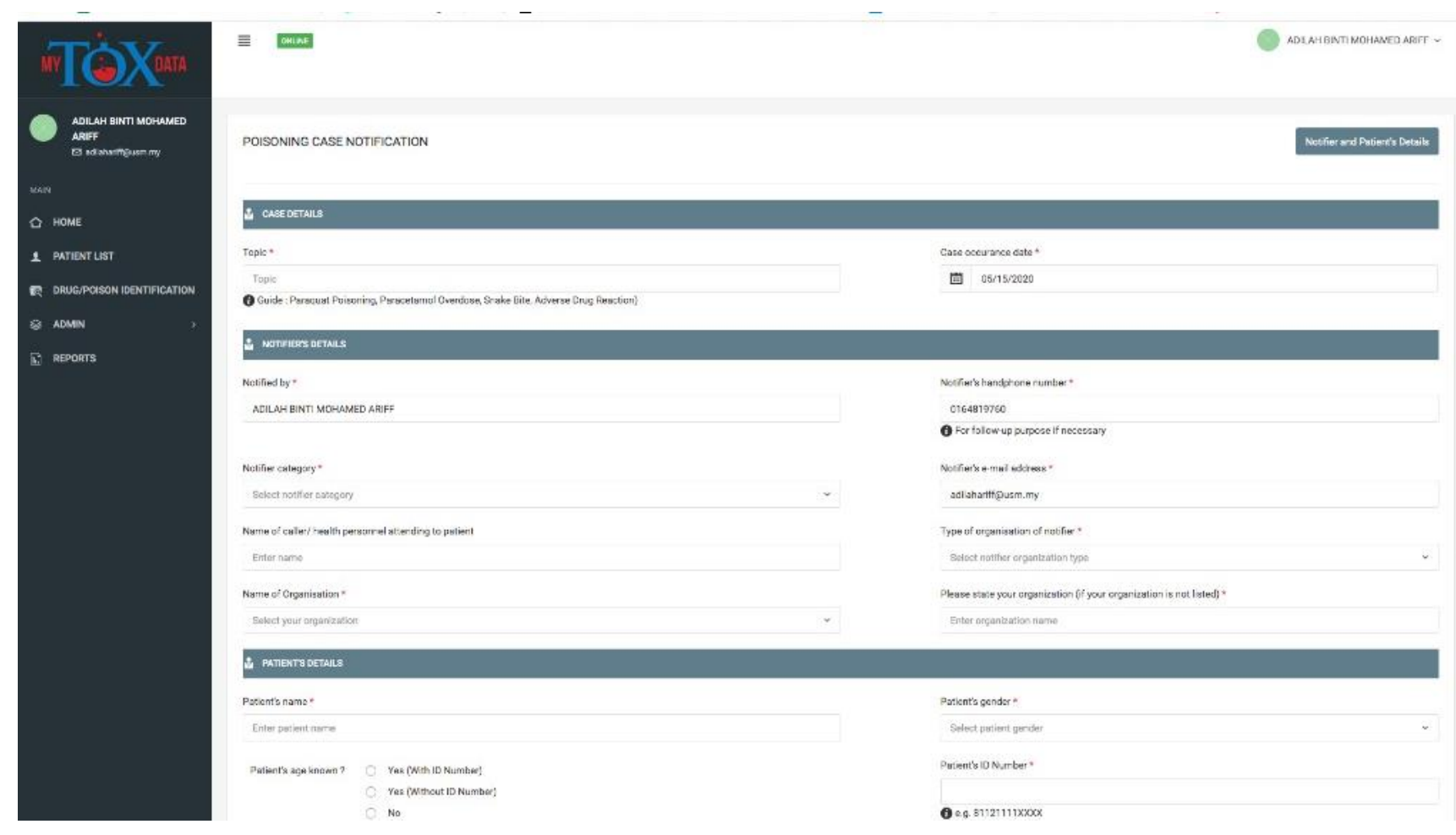

Figure 3: Case registration by the health professionals

Toxicological/poisoning case notification allows healthcare professionals to submit online poisoning notification, and the submission leads to an automated information retrieval. MyToxData captures details of notifiers who are commonly healthcare professionals and PRN staff, case details and patients' details.

Healthcare professionals and PRN staff could view the details of the listed patients and their individual status, i.e. whether they are still admitted, were discharged or died including the cause of death. Poisoning substance identification in

Figure 4 allows healthcare professionals to detect specific agents involved in poisoning cases, prior to the submission of the notification form to the PRN. The specific agents are classified as pesticide, household products, pharmaceutical and natural toxin. The uniqueness of MyToxData is that it will automatically display the information concerning a case, if such case has been submitted earlier by a health professional. 


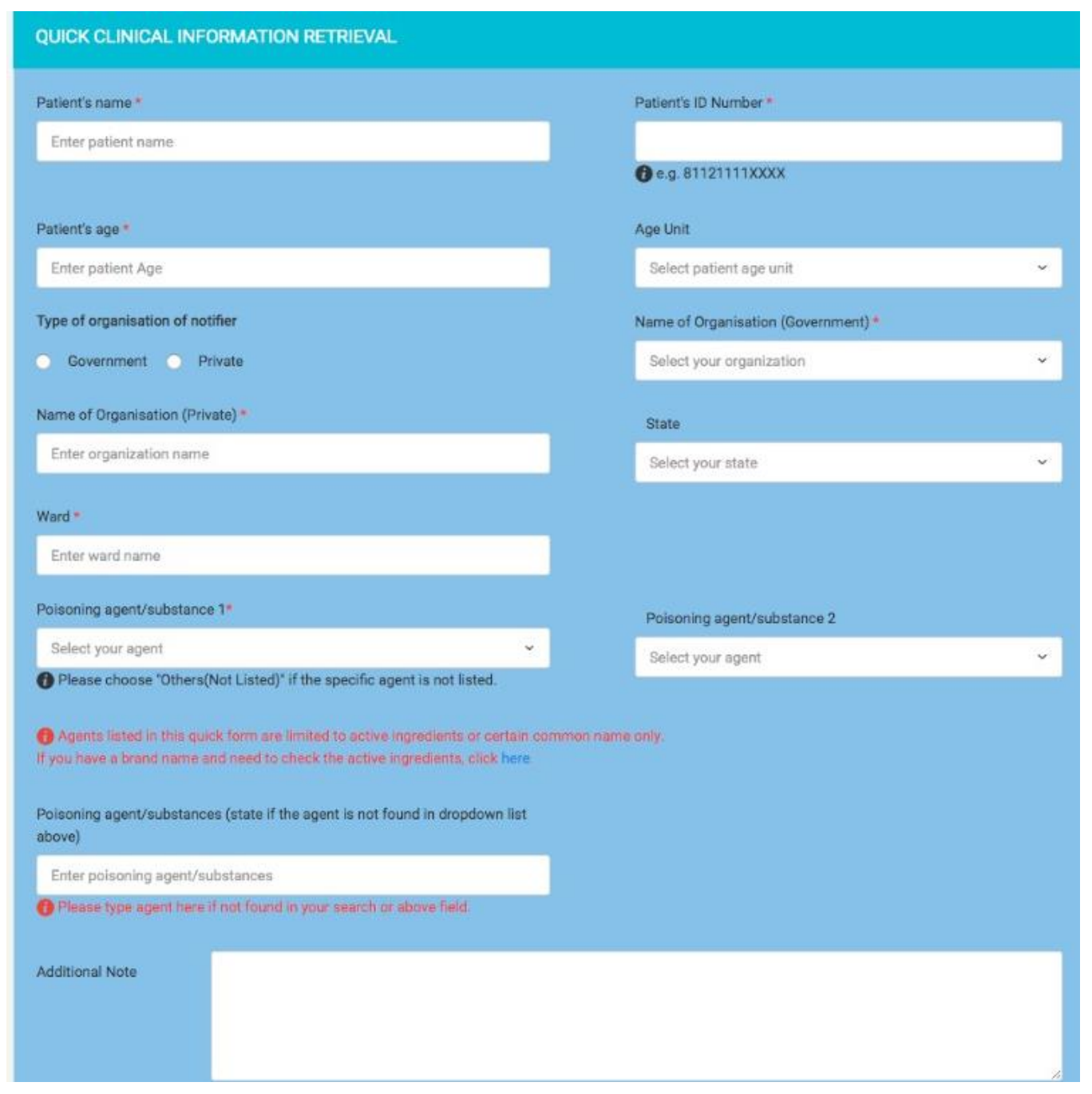

Figure 4: information retrieval for health professionals

Treatment and clinical information retrieval allow healthcare professionals to view information and toxicity of the substance implicated in a reported poisoning case. To view the required information, healthcare professionals need to login and submit an online notification form of the poisoning case. Statistical data retrieval components in Figure 5 allow users to view reported poisoning cases in the dashboard formats. Data captured in realtime can generate new knowledge and evidence to better understand patterns of poisoning cases. Both healthcare professionals and PRN staff could view the distribution of cases based on state, gender, organisation, specific agent and any data depending on the users' preferences. MyToxData integrates analytical techniques to view and report cases in a meaningful format.

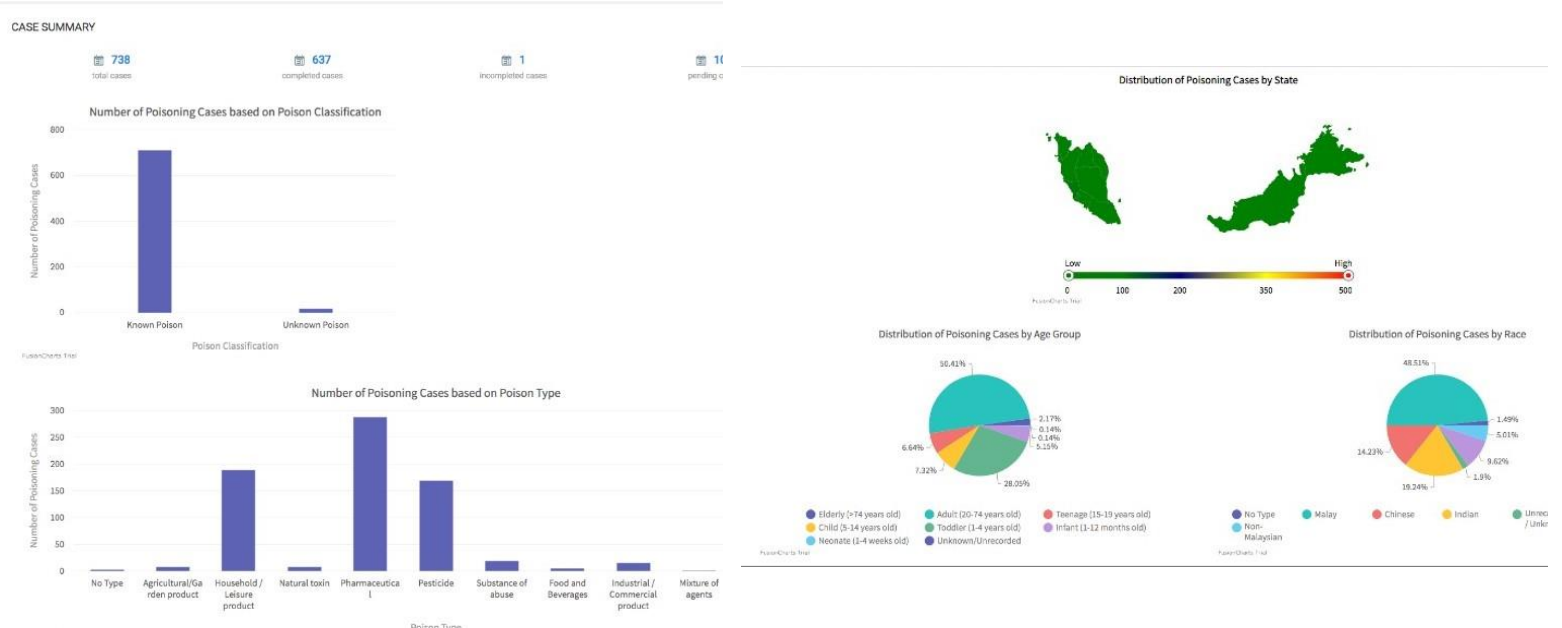

Figure 5: Statistical analysis

Realising the current toxicological issues in the country, the system was developed to enhance compliance to poisoning notification provisions in 
the related activities and regulations, harmonising poisoning data collections by various organisations to represent national epidemiological data for better understanding of the true burden and issues of poisoning in the country. Additionally, the system was established to enhance access to poisoning statistics by all relevant authorities, to enable healthcare professionals to access clinical information to manage poisoning cases and to provide a platform for the database of chemical-

based products, including pesticide, common medicine and household goods.

\section{Measurement of constructs}

This study applied a quantitative survey with a structured questionnaire. The questionnaire comprises four sections. The first section consists of user satisfaction rating on user-friendly features of all the components. Comment box is provided for the users to indicate any errors or limitations they found in the component, and they were allowed to upload the screenshot of such errors.

\section{COMPONENT 2: DRUG/POISON IDENTIFICATION}

\section{A2. Drug/Poison identification component is a very useful feature?}

\begin{tabular}{lllllll} 
& 1 & 2 & 3 & 4 & 5 & \\
\hline Strongly disagree & $\bigcirc$ & $\bigcirc$ & $\bigcirc$ & $\bigcirc$ & $\bigcirc$ & Strongly agree \\
& & \\
Found any error in this component? & & To upload a screenshot of error (if any) \\
Yes & & Browse Files \\
No
\end{tabular}

\section{Comment and suggestions (if any):}

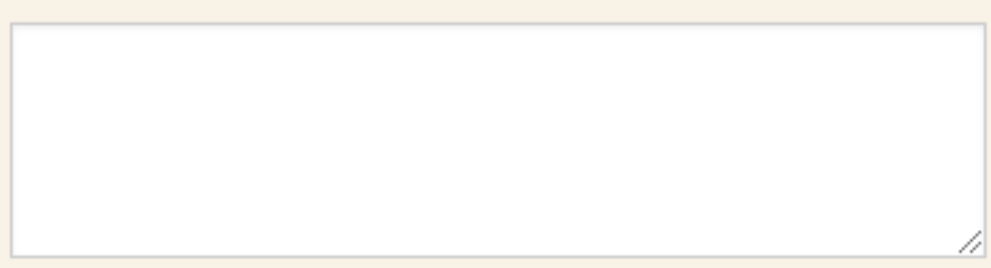

Figure 6: Screenshot of the first section of the questionnaire.

constructs of user satisfaction experience. The measurement was adopted from Doll and Torkzadeh $^{6}$, which was used to evaluate five

The second section of the questionnaire comprises the observation of the devices, operating system and internet browser utilised by the users to access MyToxData. Smartphone brand and model are also asked. The devices the users will more likely use to access MyToxData in the future are also asked. The second section is aimed to ensure that MyToxData can function well for all devices, operating system and all Internet browsers. The third section consists of the measurement of the aspects, namely, content, accuracy, format, ease of use and timeliness. Doll and Torkzadeh have compiled twelve questionnaire items and these questions were used as guidelines with necessary wording changes tailored to the proposed system. The user satisfaction rating uses a five-point scale ('very satisfied', 'satisfied', 'neutral', 'dissatisfied' and 'very dissatisfied'). The user satisfaction measurement for MyToxData is shown in

Table 1.

Table 1: Measurement of user satisfaction experience of MyToxData

\begin{tabular}{clll}
\hline Number & Construct & Item \\
\hline 1 & Accuracy & Is MyToxData accurate? & \\
\hline
\end{tabular}




\begin{tabular}{|c|c|c|}
\hline 2 & & Are you satisfied with the accuracy of MyToxData? \\
\hline 3 & Content & Does MyToxData provide the precise information you need? \\
\hline 4 & & Does the information content meet your needs? \\
\hline 5 & & $\begin{array}{l}\text { Does MyToxData provide reports that seem to be just about exactly what you } \\
\text { need? }\end{array}$ \\
\hline 6 & & Does MyToxData provide sufficient information? \\
\hline 7 & Ease of & Is MyToxData user friendly? \\
\hline 8 & use & Is MyToxData easy to use? \\
\hline $\begin{array}{c}9 \\
10\end{array}$ & Format & $\begin{array}{l}\text { Do you think the output is presented in a useful format? } \\
\text { Is the information clear? }\end{array}$ \\
\hline 11 & Timeliness & Do you get the information you need in time? \\
\hline 12 & & Does MyToxData provide up-to-date information? \\
\hline
\end{tabular}

\section{Data collection}

An online questionnaire using JotForm was distributed among healthcare professionals in Malaysian hospitals. The participants were asked to perform tasks on MyToxData system before rating their experience. Sampling was made via convenience sampling where the healthcare professionals were selected from current new users who had signed up to use the system and had been contacting the PRN for toxicological information with the rest of them selected from one of the hospitals in the same region/state of PRN. A few follow-up sessions were made via telephone calls to ensure that the users would respond to the online survey form after completing the task given to examine all the Table 2, males and females constituted almost an equal number

of study participants $\mathbf{( 5 6 . 5 \%}$ and $43.5 \%$, respectively), with a reported age distribution of 30-39 years (65.2\%). Among the present study participants, $47.8 \%$ were from a government hospital, whereas $43.5 \%$ were from other institutions. The respondents were mostly Table 3. As shown by the results, user login component has the highest agreement, i.e. $95.7 \%$, and mean $=4.35$. Case registration ranked second with $65.2 \%$ agreement and mean $=4.30$. Treatment and clinical information retrieval components ranked third with $91.3 \%$ agreement and mean $=4.22$. Toxicological $/$ poisoning case notification and poisoning substance Table 4, for MyToxData system, the main modes of access were through computer/desktop (52.2\%) and smartphone $(34.8 \%) ; 47.8 \%$ of respondents indicated that they would access MyToxData via Table 2: Healthcare Professionals' Demographic Characteristics

\begin{tabular}{lll}
\hline Variable $(\mathbf{N}=\mathbf{2 3})$ & & Frequency (\%) \\
\hline Gender & Male & $10(43.5)$ \\
& Female & $13(56.5)$ \\
Age group (years) & $20-29$ & $5(21.7)$ \\
& $30-39$ & $15(65.2)$ \\
& $49-45$ & $2(8.7)$ \\
Healthcare staff & $>45$ & $1(4.3)$ \\
& Medical specialist & $4(17.4)$ \\
& Medical officer & $9(39.1)$ \\
& Houseman & $2(8.7)$ \\
& Doctor pharmacist & $8(34.8)$ \\
\hline
\end{tabular}

components in the newly developed system.

\section{Analysis}

The collected data were then analysed using the SPSS software package version 24.0. Descriptive statistics, reliability analysis and one sample ttest were used in the present study. The factors with $\mathrm{p}$-values of less than 0.05 were considered as a significant satisfaction value.

\section{RESULTS}

\section{Respondents' Profiles}

The respondents of the present study's survey involved 23 healthcare professionals. As shown by demographic characteristics in

medical doctors (39.1\%) and pharmacists (34.8\%) with $52.2 \%$ working at emergency departments. Generally, they had working experience of over 5 years $(65 \%)$.

\section{Components Feedback}

Responses to survey items asked in the first section are shown in

identification received moderate agreement from the respondents with mean $=3.61$ and 3.48, respectively.

\section{Accessing Devices}

As shown in

smartphone and laptop/desktop in future, and 69.9\% accessed the system via Google Chrome browser. 
Malaysian Journal of Public Health Medicine 2021, Vol. 21 (2): 234-242

\begin{tabular}{lll}
\hline Organisation & Government hospital & $11(47.8)$ \\
& District hospital & $2(8.7)$ \\
Practice site & Others & $10(43.5)$ \\
& Emergency department & $12(52.2)$ \\
& Medical department & $1(4.3)$ \\
Working experience & Pharmacy department & $2(8.7)$ \\
& Others & $8(34.8)$ \\
& Less than 5 years & $8(34.8)$ \\
\hline
\end{tabular}

Table 3: Rating assessment of MyToxData

\begin{tabular}{llllllll}
\hline Variable $\mathbf{( N ~ = ~ 2 3 ) ~}$ & $\begin{array}{l}\text { Strongly } \\
\text { disagree } \\
(\%)\end{array}$ & $\begin{array}{l}\text { Disagree } \\
\mathbf{( \% )}\end{array}$ & $\begin{array}{l}\text { Neutral } \\
\mathbf{( \% )}\end{array}$ & $\begin{array}{l}\text { Agree } \\
\mathbf{( \% )}\end{array}$ & $\begin{array}{l}\text { Strongly } \\
\text { agree } \\
(\%)\end{array}$ & Mean & Remark \\
\hline $\begin{array}{l}\text { User login } \\
\text { Case registration }\end{array}$ & - & 4.3 & - & 52.2 & 43.5 & 4.35 & High: 1 \\
$\begin{array}{l}\text { Poisoning substance } \\
\text { identification }\end{array}$ & - & 4.3 & 17.4 & 21.7 & 56.5 & 3.48 & Moderate: \\
$\begin{array}{l}\text { Treatment and clinical } \\
\text { information retrieval }\end{array}$ & - & 4.3 & 4.3 & 56.5 & 34.8 & 4.22 & High: 3 \\
$\begin{array}{l}\text { Toxicological/poisoning } \\
\text { case notification }\end{array}$ & 13.0 & 8.7 & 13.0 & 34.8 & 30.4 & 3.61 & Moderate: \\
\hline
\end{tabular}

Table 4: Type of devices and browsers used to access MyToxData

\begin{tabular}{lll}
\hline Variable $(\mathbf{N}=\mathbf{2 3})$ & Frequency (\%) \\
\hline Current access device & Smartphone & $8(34.8)$ \\
& Tablet & $3(13.0)$ \\
Future access device & Laptop/desktop & $12(52.2)$ \\
& Smartphone & $11(47.8)$ \\
Browser device & Tablet & $1(4.3)$ \\
& Laptop/desktop & $11(47.8)$ \\
& Google Chrome & $16(69.6)$ \\
& Internet Explorer & $1(4.3)$ \\
& Mozilla Firefox & $2(8.7)$ \\
& Safari & $2(8.7)$ \\
\hline
\end{tabular}

\section{Satisfaction Assessment}

With a coefficient alpha of more than 0.89 for all constructs, the result of the measurement was found to be reliable. The satisfaction questionnaire could be understood by the respondents, and given the reliability benchmark,

Table 5, respondents are significantly satisfied

Table 5: Satisfaction assessment of MyToxData the respondents were satisfied with the system ${ }^{7}$.

One sample t-test was performed to measure MyToxData system satisfaction based on five constructs, namely, accuracy, content, ease of use, format and timeliness. As shown in with MyToxData system based on all the five constructs.

\begin{tabular}{|c|c|c|c|c|c|}
\hline & \multirow[t]{2}{*}{$\mathrm{t}$} & \multirow[t]{2}{*}{$\mathrm{p}$-value } & \multirow[t]{2}{*}{ Mean difference } & \multicolumn{2}{|c|}{$95 \%$ Confidence interval of the difference } \\
\hline & & & & Lower & Upper \\
\hline Accuracy & 34.834 & $<.01$ & 4.3261 & 4.069 & 4.584 \\
\hline Content & 27.365 & $<.01$ & 4.2065 & 3.888 & 4.525 \\
\hline $\begin{array}{l}\text { Ease of } \\
\text { use }\end{array}$ & 18.887 & $<.01$ & 3.8913 & 3.464 & 4.319 \\
\hline Format & 27.887 & $<.01$ & 4.1522 & 3.843 & 4.461 \\
\hline
\end{tabular}




$\begin{array}{lllll}\text { Timeliness } & 22.394 & <.01 & 3.511 & 3.8696\end{array}$

\section{DISCUSSION}

The present study was conducted in response to the lack of an organisational system that caters the needs of toxicovigilance in Malaysia. Consequently, MyToxData system was developed to serve as a resource for management and enhancement of toxicology-related services in Malaysia. The present study, which involved 23 healthcare professionals, has revealed some interesting data on the use of the proposed system.

Based on the evaluation of the MyToxData system user components, most of the respondents (95.7\%) reported that the user login was user friendly, $65.2 \%$ of whom were healthcare professionals who agreed that the registration and toxicological/poisoning case notification through the system was straightforward. The poisoning substance identification component received a $78.2 \%$ agreement on the usefulness of this feature in minimising their time while using the system. Furthermore, the present study showed that 91.3\% agreed that the treatment and clinical information retrieval made available in this system are useful. These results supports the findings that shows users' satisfaction is very important in system adoption ${ }^{8}$.

Disagreements recorded among the respondents in terms of usefulness and ease of use of certain components of the system indicates the need to review and enhance such components accordingly to meet user satisfaction and increase their inclination to use the system in the future. Case registration is one of the main components recorded to receive negative feedback the most, with $8.7 \%$ disagreement and $13 \%$ strong disagreement. In reporting a toxicological case, submitting all relevant data of the incident are essential to ensure that the identity of the substance involved relates directly to the patient's clinical information, and capturing sufficient demographic data will facilitate toxicovigilance-related decisions. Considering this factor, the registration form of each case was initially designed with five basic sections, namely, case details, patient's details, agent information, incident details and risk and treatment section. The amount of the data necessary to be entered may have caused

negative feedback from the respondents. After completing this study, the toxicological case report form is modified, and a quick form version is developed as an alternative for those who need quick treatment and clinical information retrieval from the system.
Toxicological/poisoning case notification component was found to receive negative feedback the same as case registration. This component can only be tested after a poisoning case was registered in the system. Relatively, this component consists of two very simple questions or outcome information form that users have to fill-up to update a patient's outcome after discharge from the health facility. The negative feedback may be due to that this component was not found to be updated, was not able to follow instructions from the research team, or was not tested during the trial session.

The system was predominantly accessed using non-handheld devices via Google Chrome (69.6\%), highlighting a limited use of smart devices with only $34.8 \%$ accessed the system on their smartphones. However, $47.8 \%$ showed an inclination to access the system using a handheld smart device in future. This finding may be due to practicality and ease of access during actual practise and application especially in poisoning emergency setting. Moreover, $52.2 \%$ of the respondents mainly practise in the Emergency Department.

The reliability results have shown that the questionnaire used is valid and reliable for endusers of toxicological information system. Results of one sample t-test showed that MyToxData system achieves significant satisfaction levels in terms of accuracy, content, ease of use, format and timeliness. The findings of this research was consistent with $^{(6)}$. The findings show that the system could be viewed as one of the most promising ways to enhance toxicological cases, including case registration, toxicological/poisoning case notification, poisoning substance identification, treatment and clinical information retrieval. This suggests that the proposed and developed MyToxData system by the PRN supports the digital healthcare and creating a sustainable system improved toxicological data sharing, advanced analytics and automation.

\section{CONCLUSIONS}

The only limitation of the present study was the number of institutions involved, which may have affected the overall generalisability of the outcome. The present study, however, has provided a much-needed toxicological information system for collecting, managing and reporting the toxicological data in poison centres. The five satisfaction constructs of the MyToxData have been significantly identified as effective features of the system by a vast majority of the healthcare professionals. Hence, the results have indirectly shown that toxicological system could be adopted by the healthcare insiders. The system 
effectiveness could add value to the poison centres in enhancing productivity, thereby making toxicological management decisions better informed while allowing for significant gains in efficiency and cost control.

\section{Acknowledgement}

The authors would like to thank Enago (www.enago.com) for the English language review.

\section{Disclosure statement}

The authors report no conflict of interest.

\section{Funding Information}

This work was supported by the Universiti Sains Malaysia, Malaysia under Research University Grant (1001/PPAMC/8012234).

\section{Complying with Ethics of Experimentation}

This study follows the principles of the Declaration of Helsinki.

\section{REFERENCES}

1. Tangiisuran B, Jiva M, Ariff AM, Rani NAA, Misnan A, Rashid SM, et al. Evaluation of types of poisoning exposure calls managed by the Malaysia National Poison Centre (2006-2015): a retrospective review. BMJ Open. 2018;8(12):e024162.

2. Rajasuriar R, Awang R, Hashim $S B H$, Rahmat HRBH. Profile of poisoning admissions in Malaysia. Human \& Experimental Toxicology. 2007;26(2):7381.

3. World Health Organization. IPCS INTOX. World Health Organization; 2013.

4. Aggelidis VP, Chatzoglou PD. Hospital information systems: Measuring end user computing satisfaction (EUCS). Journal of biomedical informatics. 2012;45(3):56679.

5. Ferreira JM, Acuña ST, Dieste O, Vegas S, Santos A, Rodríguez F, et al. Impact of usability mechanisms: An experiment on efficiency, effectiveness and user satisfaction. Information and Software Technology. 2020;117:106195.

6. Doll WJ, Torkzadeh G. The measurement of end-user computing satisfaction. MIS quarterly. 1988:259-74.

7. Greco LM, O'Boyle EH, Cockburn BS, Yuan Z. Meta-analysis of coefficient alpha: A reliability generalization study. Journal of Management Studies. 2018;55(4):583-618.

8. Verma P, Kumar S, Sharma SK. eHealthcare service quality: consumer satisfaction and its association with demographic characteristics. International Journal of Health Care Quality Assurance. 2020. 